\title{
RUNX3 methylation and anti-tumor immunity
}

Editorial

\section{Trevelyan R. Menheniott, Louise M. Judd and Andrew S. Giraud}

Comment on: Lineage-specific RUNX3 hypomethylation marks the preneoplastic immune component of gastric cancer. Oncogene. 2015; 34:2856-66.

Altered DNA methylation is a hallmark of cancer genomes and has been advocated as both a marker and mechanism of the malignant phenotype. In the conventional view, such alterations include global DNA hypomethylation, linked to aberrant expression of oncogenic driver genes and more localised de novo hypermethylation at 'CpG-island' promoters, argued to cause inappropriate silencing of tumor suppressor genes. Although paradigms like these have been unquestionably influential, deregulation of the epigenome is not the only source of altered DNA methylation in tumors. Cell lineage and tissue composition have recently emerged as additional determinants of cancer-specific methylation patterns $[1,2]$.

During differentiation, cell lineages acquire stable and heritable gene expression programs associated with characteristic epigenetic marks, including DNA methylation. Lineage-specific DNA methylation profiles can therefore serve as 'epigenetic fingerprints' of cellular identity, suggesting their application as diagnostic biomarkers for diverse cell types. Screening for subliminal lineage signatures concealed within 'DNA methylomes' may allow simultaneous detection and enumeration of multiple cell types in complex tissues [3], including cancers [2]. Indeed, methylation signatures reflecting the presence of key prognostic indicators, such as $\mathrm{T}$ lymphocytes, have already shown immense promise in predicting clinical outcome of cancers and response to therapy [2].

Gastric cancer (GC), which has one of the highest malignancy-related mortality rates globally, arises through stepwise, inflammation-dependent transformation of gastric epithelial cells. Improved detection of the premalignant inflammatory stages of GC may facilitate earlier intervention and better outcomes [4]. In a recent study by Kurklu et al. [5] we described an aberrant hypomethylation signature at the RUNX3 locus, associated with GC progression, specifically within an upstream alternative promoter (RUNX3 P1). We found that, based on the degree of $R U N X 3 \mathrm{P} 1$ hypomethylation, premalignant gastric epithelial lesions and tumors could be segregated from disease-free tissues. This novel signature may therefore be diagnostic for premalignant inflammation in individuals who are, by definition, at risk of developing GC.

Importantly, our analysis utilised whole tissues/ tumors comprised primarily by cancer epithelial cells, but also by non-neoplastic cell types including leukocytes. It had not escaped our attention that $R U N X 3$, a key regulator of haematopoiesis, is highly expressed in several immune cell lineages [6]. We therefore hypothesised that enrichment of $R U N X 3 \mathrm{P} 1$ hypomethylated alleles in these tissues might reflect leukocyte recruitment to the premalignant epithelium and/or tumor microenvironment. DNA methylation analysis of epithelial and immune cell lineages independently isolated directly from gastric tumors confirmed this expectation; RUNX3 P1 was hypermethylated and repressed in the epithelial cells but, critically, lacked methylation and was transcriptionally active in all the isolated immune cell types tested. Therefore, $R U N X 3 \mathrm{P} 1$ hypomethylation, as a 'pan'-marker of haematopoietic lineages, can distinguish progressive changes in cellular composition that accompany GC pathogenesis, namely those due to leukocyte infiltration (Figure 1).

A key question not addressed in our study is whether RUNX3 P1 methylation, as a surrogate of leukocyte influx, might classify gastric tumors into useful prognostic categories? As a case in point, lymphocyterich GC generally has a better prognosis than GC lacking significant lymphocytic infiltrate [7]. Intra-tumoral lymphocytes often display cytolytic properties positively linked to survival, hence defining specific methylation signatures of cancers that elicit this type of anti-tumor response would be of high translational priority. The value of such an approach has been shown to great effect in breast cancer. Dedeurwaerder et al. described a multigene methylation signature that could not only discern the degree of tumor infiltration by $\mathrm{T}$ lymphocytes, but was also strongly prognostic for relapse-free survival [2]. They concluded that their signature was likely indicative of an anti-tumor T cell response. While the jury is still out, $R U N X 3$ P1 methylation has links with key mediators of anti-tumor responses that warrant exploration of its use in similar context. For example, RUNX3 P1 hypomethylation is a feature of cytolytic CD8+ T lymphocytes and natural killer cells (NK) [5], correlating with both the high $R U N X 3$ mRNA expression of these two cell types and genetic requirement of $R U N X 3$ for their intrinsic 'tumor killing' functions [6]. Nevertheless, we caution against over interpretation of these observations, as RUNX3 $\mathrm{P} 1$ hypomethylation also marks other immune lineages that can elicit both pro- and anti-tumor effects. Ultimately, 
A
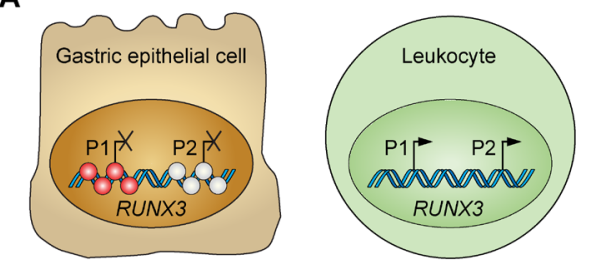

Methylated $\mathrm{CpG}(5 \mathrm{mC})$

Repressive histones

B

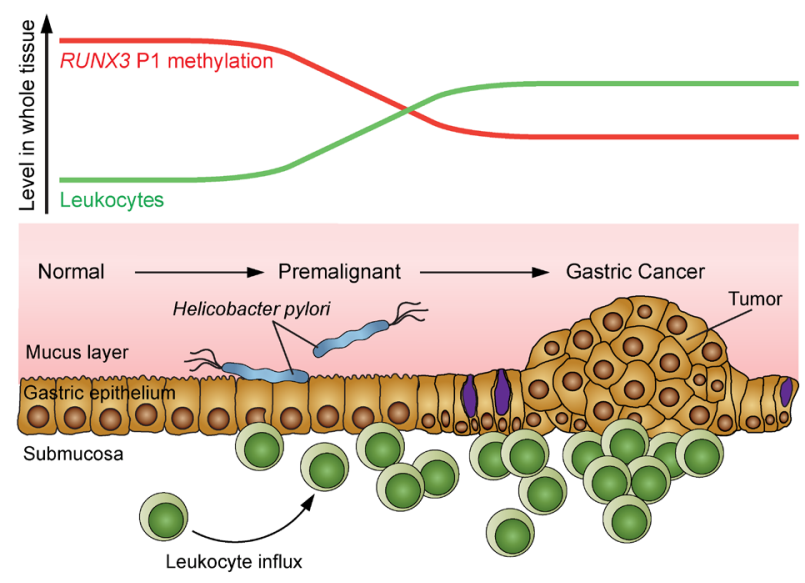

Figure 1: RUNX3 P1 methylation levels reflect leukocyte infiltration during GC progression. A. $R U N X 3 \mathrm{P} 1$ shows differential DNA methylation (5'methyl cytosine; $5 \mathrm{mC}$ ) between gastric epithelial cell and leukocytes. $R U N X 3 \mathrm{P} 2$, like most $\mathrm{CpG}$ island promoters in normal cells, is controlled by histone modification, not DNA methylation. B. Normal (disease-free) gastric epithelium lacks inflammation. Premalignant inflammation (triggered by Helicobacter pylori infection) and subsequent tumor growth is characterised by progressive leukocyte infiltration. This change in cell type composition is signified by a corresponding decrease in RUNX3 P1 methylation levels.

whether RUNX3 P1 methylation can reveal the specific polarity of cancer immune responses, used alone or in combination, awaits formal validation in appropriately powered clinical studies.

We are not the first to study RUNX3 methylation as a cancer biomarker. Aberrant hypermethylation of the canonical RUNX3 $\mathrm{CpG}$ island promoter (RUNX3 P2) hitherto proposed [8], but since refuted as a driver of GC has been avidly pursued as a biomarker, albeit with limited success (reviewed in [6]). However, our analysis of $R U N X 3 \mathrm{P} 1$ is unique in the field, providing fresh perspectives on cell lineage as a modifier of cancer methylomes and further highlighting its potential for translation in GC as well as other malignancies in which leukocyte infiltration has diagnostic and prognostic significance.

\section{CONFLICT OF INTEREST}

The authors declare that there are no conflicts of interest associated with this publication.

Trevelyan R. Menheniott: Murdoch Children's Research Institute, Department of Paediatrics, University of Melbourne, Melbourne, Victoria, Australia
Correspondence: Trevelyan R. Menheniott, email treve. menheniott@mcri.edu.au

Keywords: cancer, DNA methylation, epigenetics, RUNX3, anti-tumor immunity

Received: August 06, 2015

Published: September 12, 2015

\section{REFERENCES}

1. Sproul D, et al. Proc Natl Acad Sci U S A. 2011; 108:4364-4369.

2. Dedeurwaerder S, et al. EMBO Mol Med. 2011; 3:726-741.

3. Accomando WP, et al. Genome Biol. 2014; 15:R50.

4. Correa P. Gastroenterol Clin North Am. 2013; 42:211-217.

5. Kurklu B, et al. Oncogene. 2015; 34:2856-2866.

6. Lotem J, et al. Biochim Biophys Acta. 2015; 1855:131-143.

7. Grogg KL, et al. Mod Pathol. 2003; 16:641-651.

8. Li QL, et al. Cell. 2002; 109:113-124. 